\title{
Pathophysiology of Pulmonary Circulation in Congenital Heart Disease
}

\author{
Megumi Hashimoto, Masahiro Kaneko, Naohisa Nishida, \\ Hina Serizawa, Kohei Kawata, Rika Sekiya, \\ and Hideaki Senzaki
}

\begin{abstract}
There are several types of abnormalities in the integrated physiology of pulmonary circulation in congenital heart disease. The main pathology of Eisenmenger syndrome involves a change in pulmonary resistance and is the most commonly observed pathophysiology in pulmonary hypertension. Other diseases also present with the main pathophysiological characteristic of reduced pulmonary compliance, such as tetralogy of Fallot and multiple peripheral pulmonary stenosis. In addition, the cavo-pulmonary connection has the unique feature of both pulmonary circulation and regulation. According to the differences in the pathophysiological features of pulmonary circulation, therapeutic approaches may considerably differ between diseases and conditions. Physiology-based clinical insights with regard to pulmonary circulation in congenital heart disease will be discussed in this chapter.
\end{abstract}

\section{Keywords}

Impedance $\cdot$ Wave reflection $\cdot$ Characteristic impedance $\cdot$ Compliance $\cdot$ Pressurevolume relationships

M. Hashimoto $\cdot$ M. Kaneko $\cdot$ N. Nishida $\cdot$ H. Serizawa $\cdot$ K. Kawata

R. Sekiya $\cdot$ H. Senzaki $(\bowtie)$

Pediatric Cardiology and Pediatrics, Kitasato University School of Medicine,

Kanagawa, Japan 


\subsection{Introduction}

This chapter discusses the pathophysiology of pulmonary circulation in congenital heart disease. This refers to the macrophysiology of cardiovascular hemodynamics, an understanding of which is essential for the treatment of patients with abnormal pulmonary circulation and congenital heart disease. We hope this chapter can provide some hints and insights for use in future research on pulmonary hypertension in congenital disease as well as in the fields of physiology and molecular and cellular biology.

We will first discuss how we comprehensively assess integrated pulmonary circulation in congenital heart disease in vivo. Subsequently, using methodology for comprehensive assessment, we will discuss the pathophysiological characteristics of pulmonary circulation in patients with various types of congenital heart disease.

\subsection{Comprehensive Assessment of Integrated Pulmonary Circulation}

\subsubsection{Physiologic Components of Pulmonary Circulation}

Pulmonary circulation consists of the following three major components: resistive, elastic, and reflective components. The resistive component is described as pulmonary vascular resistance (PVR). PVR acts in opposition to mean or non-pulsatile steady flow and is analogous to electric resistance, which acts in opposition to direct current in an electronic circuit. Thus, PVR can be calculated as the ratio of mean pulmonary flow to mean pulmonary arterial pressure according to Ohm's law for an electric circuit. In other words, PVR only determines mean pulmonary pressure according to the amount of pulmonary blood flow. An increase in PVR causes an increase in mean pulmonary arterial pressure without a marked change in pulse pressure (Fig. 15.1a).

The elastic component is vascular compliance or capacitance. The pulmonary artery is not a rigid tube but has elasticity to buffer intermittent pulsatile flow ejected by the right ventricle. Pulmonary arterial capacitance is a vascular property in this buffering system and is determined by both arterial wall elasticity and vascular size or volume. Pulmonary compliance/capacitance is analogous to a capacitor or condenser in an electric circuit. An alternating current but not a direct current goes in and out of the capacitor. Similarly, only pulsatile flow goes in and out of the pulmonary vascular bed with capacitance and is associated with a change in pulse pressure. When pulmonary capacitance is decreased, in other words, when the pulmonary arterial wall is stiff and/or pulmonary vascular bed is small, blood pressure increases through an increase in pulse pressure without a change in mean blood pressure (Fig. 15.1b).

There is yet another property of the elastic component of the pulmonary vascular bed. This is termed characteristic impedance and represents wall stiffness in the proximal artery. In an electric circuit, characteristic impedance is described as 
a

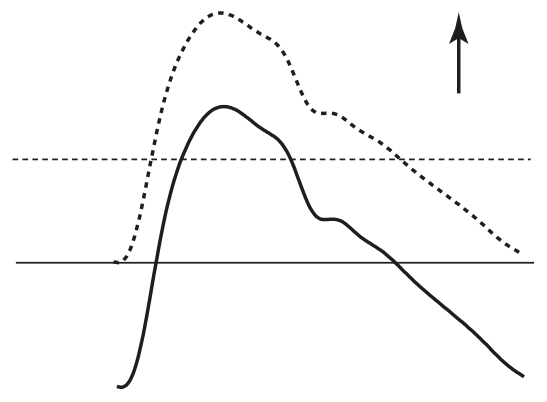

C

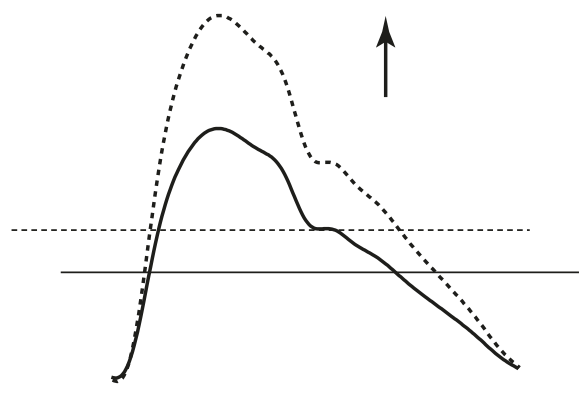

b

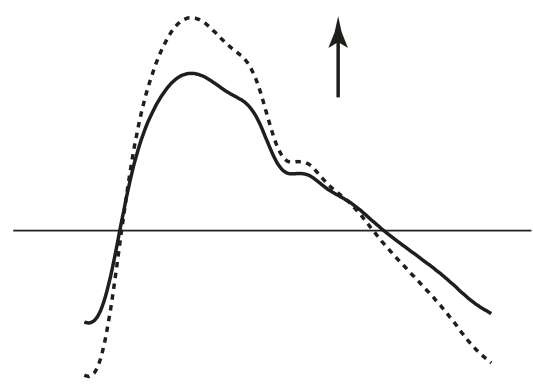

d

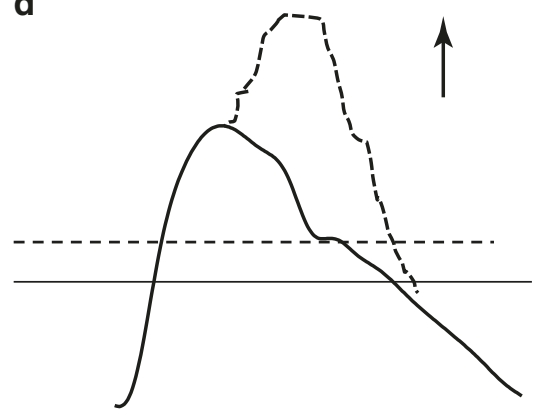

Fig. 15.1 Effects of changes in resistance (a), compliance (b), characteristic impedance (c), and wave reflection (d) on pulmonary artery pressure

resistance upstream of the resistor-capacitor parallel circuit. When characteristic impedance increases, in other words, when the proximal pulmonary arterial wall stiffens, blood pressure increases by an increase in pulse pressure above the diastolic pressure as shown in Fig. 15.1c.

The last major component is a reflective component. The pulmonary vascular bed is not an infinite system but has an end at the left atrium. The pulmonary vascular bed also has many bifurcations with caliber changes. In this type of system, blood flow and pressure waves produce reflections at the site of a bifurcation or vessel caliber change [1,2]. Therefore, the measured pressure or flow we observe is the sum of forward and reflected waves, and thus augmentation of reflected waves causes significant pressure elevation (Fig. 15.1d).

Therefore, abnormalities in each component contribute differently to pressure elevation in patients with pulmonary hypertension. Resistance change increases pulmonary arterial pressure (PAP) by increasing mean arterial pressure, changes in capacitance and characteristic impedance increase arterial pressure by increasing pulse pressure, and wave reflection increases PAP by adding reflected pressure. Therefore, to better understand the pathophysiology of pulmonary hypertension, it is very important to identify to what extent each of these components is abnormal and contributes to pressure elevation in pulmonary hypertension. 


\subsubsection{Impedance Analysis}

Impedance analysis is an ideal method for this purpose by providing comprehensive information regarding the vascular system, including vascular compliance and wave reflection as well as vascular resistance [3, 4]. To briefly explain (Fig. 15.2), instantaneously measured pressure and flow data are mathematically transformed as the sum of pressure and flow waves at each frequency of the integer multiple of a fundamental frequency. The fundamental frequency corresponds to the heart rate. The ratio of pressure to flow at each frequency yields impedance spectra. Fig. 15.3 shows examples of impedance spectra. Impedance at 0 frequency, or zero oscillation, represents pulmonary resistance. Characteristic impedance is calculated as the average impedance at higher frequencies. Compliance is calculated from lower frequency impedance or the time constant of diastolic pressure decay. Wave reflection increases oscillation of the impedance spectra at higher frequencies and is directly calculated using the formula described in Fig. 15.3. Thus, all other vascular components, including characteristic impedance, pulmonary arterial compliance, and wave reflection, are obtained from these spectra.
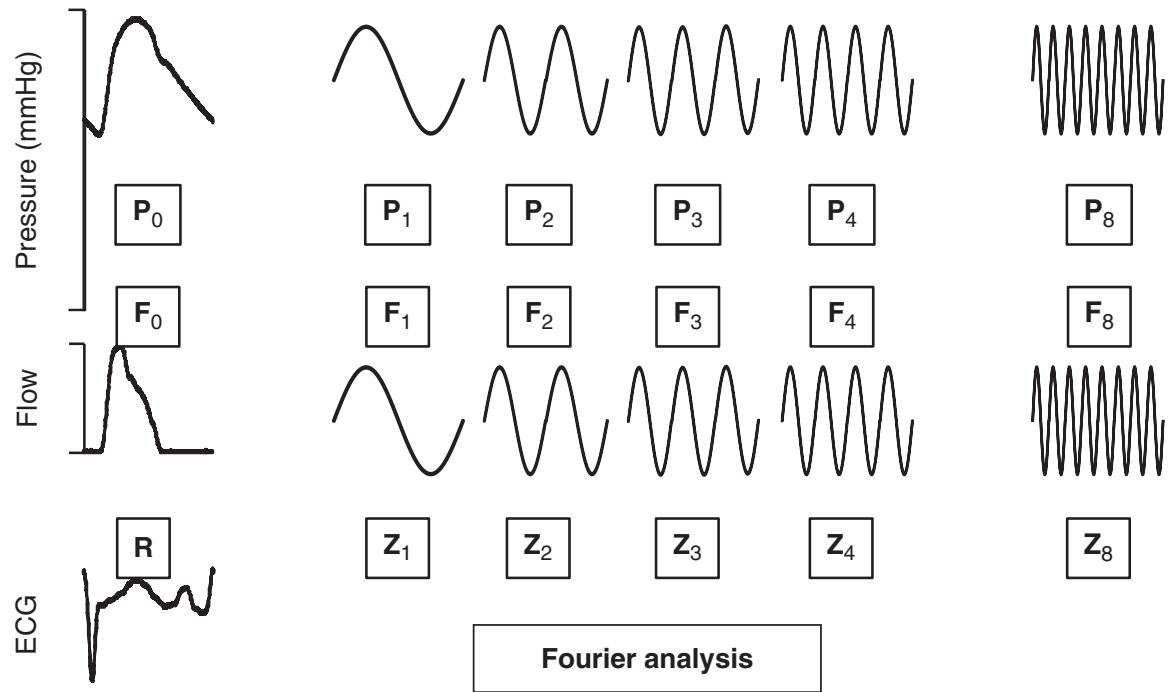

Fig. 15.2 Fourier transformation of pressure and flow data, yielding impedance spectra in the frequency domain 


\section{Impedance}

Pulmonary vascular resistance $(\mathrm{Rp})$

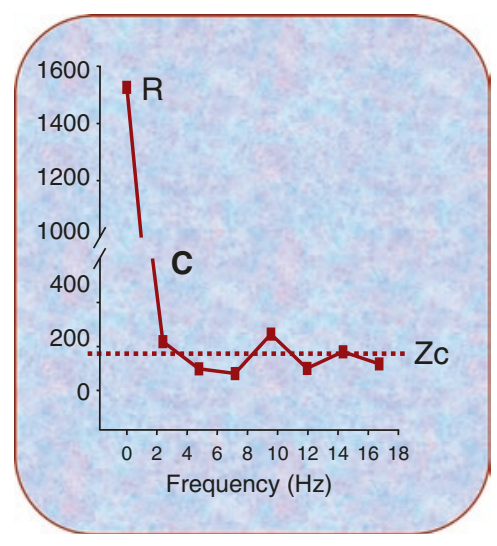

impedance at $0 \mathrm{~Hz}$ (no oscillation)

Characteristic impedance $(Z c)$

average of impedance at higher $\mathrm{Hz}$

Pulmonary arterial compliance (C)

lower $\mathrm{Hz}$ impedance

Wave reflection

$$
\mathrm{Pb}=(\mathrm{Pm}-\mathrm{Zc} \mathrm{Fm}) / 2
$$

Pm and Fm: measured pressure and flow

Fig. 15.3 Example of impedance spectra, which provide comprehensive measurements of vascular properties, including pulmonary vascular resistance, characteristic impedance, pulmonary arterial compliance, and wave reflection

\subsection{Pathophysiological Characteristics of Pulmonary Circulation in Congenital Heart Disease}

Using impedance analysis, let us discuss the pathophysiological characteristics of pulmonary circulation in patients with various types of congenital heart disease.

\subsubsection{Abnormal Resistance Is the Main Pathophysiology}

In the disease entity of abnormal pulmonary circulation, increased pulmonary resistance is the main pathophysiology. Eisenmenger syndrome due to high pulmonary flow and idiopathic pulmonary arterial hypertension (PAH) are examples. Fig. 15.4 shows an example of PAH due to patent ductus arteriosus in a 1-year-old patient with trisomy 21 . PAP is $80 / 40 \mathrm{mmHg}$. Compared to a control patient with normal pulmonary arterial pressure, wave reflection (shown via the dashed lines) is augmented in this patient. Impedance spectra exhibit an upward shift in this patient, particularly at 0 frequency or pulmonary resistance. There are also mild abnormalities in characteristic impedance $(\mathrm{Zc})$, compliance $(\mathrm{C})$, and wave reflection. In this patient, oral administration of sildenafil markedly reduced PAP as shown by the yellow lines. This is mainly the result of reduction of pulmonary vascular resistance, with some degree of improvement in vascular compliance and wave reflection. 


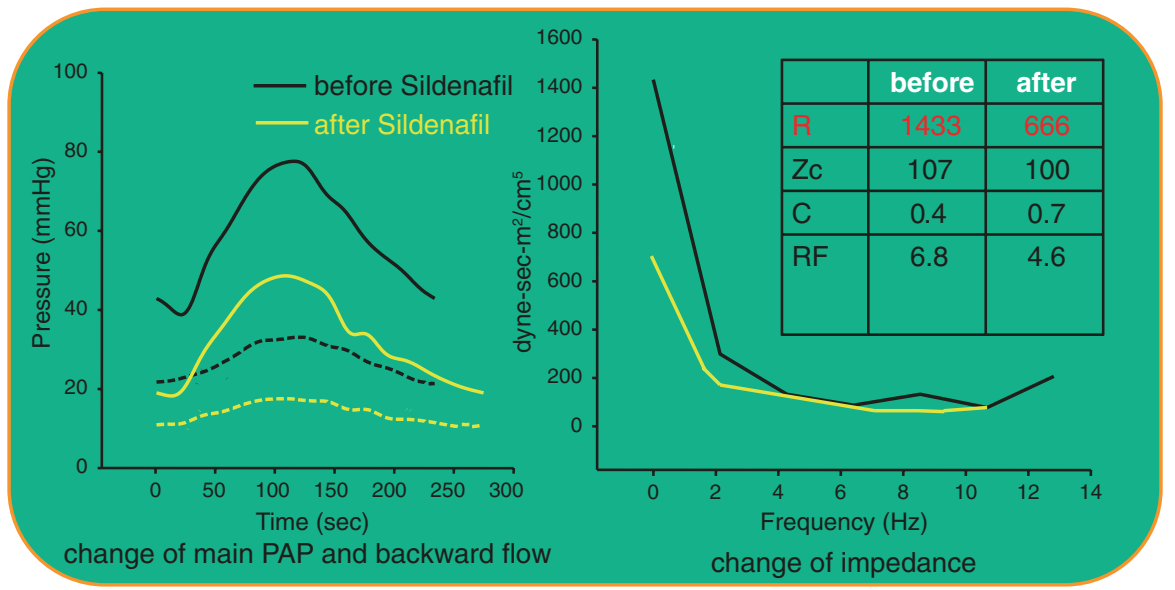

Fig. 15.4 Pressure (left) and impedance (right) data before and after oral administration of sildenafil. R, Zc, C, and RF represent resistance, characteristic impedance, compliance, and reflection factor

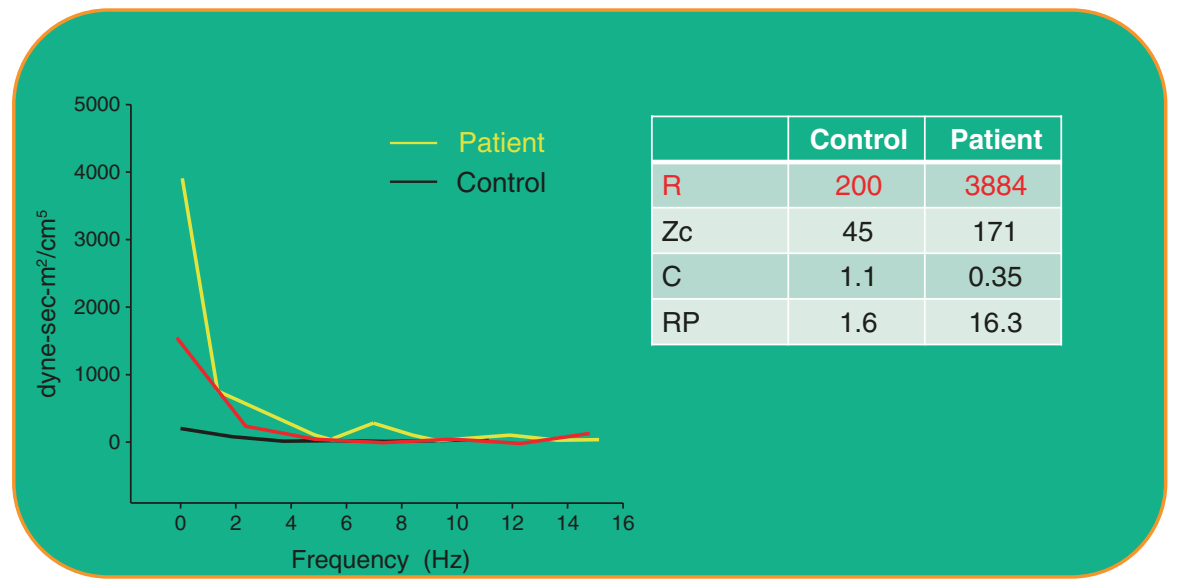

Pulmonary artery impedance

Fig. 15.5 Impedance data before and after oral administration of sildenafil. R, Zc, C, and RF represent resistance, characteristic impedance, compliance, and reflection factor

Here is another example of PAH in a 9-year-old girl after repair of transposition of the great arteries. PAP is $125 / 68 \mathrm{mmHg}$. Impedance spectra in this patient are characterized by a further upward shift of the yellow line compared to those in controls and the previous case of PAH, indicating a more advanced stage of PAH (Fig. 15.5). Interestingly, oral administration of sildenafil, as shown by the yellow line, did not have a marked effect on pulmonary vascular resistance but still reduced PAP by $20 \mathrm{mmHg}$. This was mainly owing to the change in characteristic impedance, which caused the reduction of pulse pressure (Fig. 15.6). These data show that 


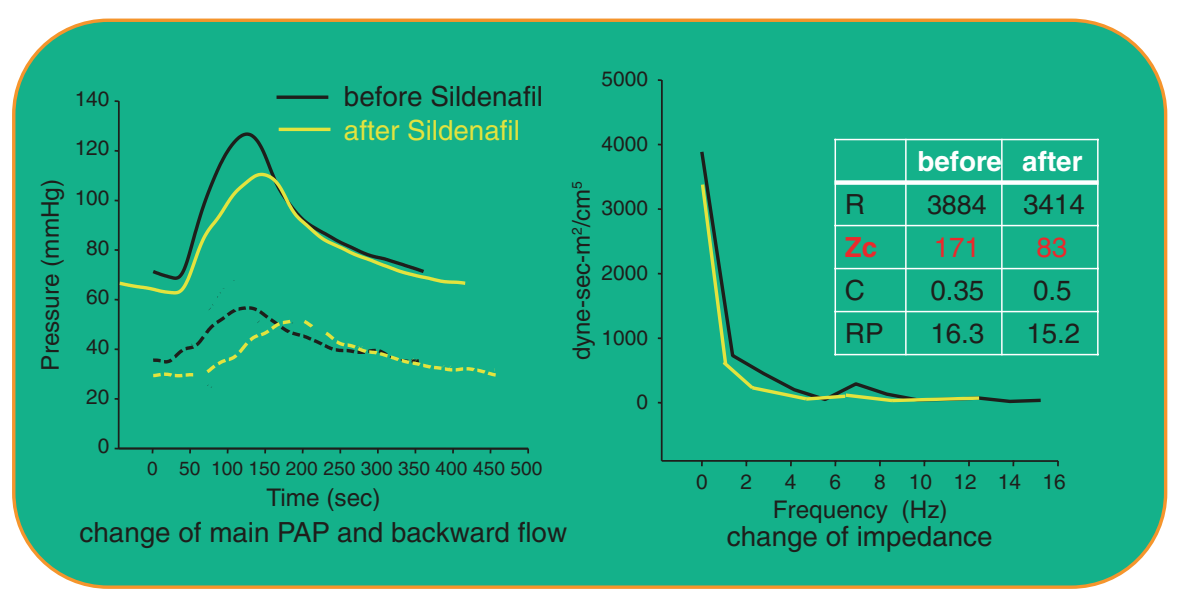

Fig. 15.6 Pressure (left) and impedance (right) data before and after oral administration of sildenafil. R, Zc, C, and RF represent resistance, characteristic impedance, compliance, and reflection factor

pharmacologic effects and the mechanism of action of pulmonary dilators can be different according to the disease stage and condition and that impedance analysis enables detailed assessment about where the main lesions of pathologic change are and how the vascular bed responds to medications.

In addition to each parameter of impedance, combining resistance and compliance as their product, RC, also provides useful information about the pulmonary circulation. $\mathrm{RC}$ represents the time required for diastolic pressure decay, and a longer $\mathrm{RC}$ indicates that the blood does not go through the pulmonary vascular bed smoothly [5].

Therefore, advanced PAH should be associated with higher RC values. In fact, although PVR alone could not clearly distinguish pulmonary hypertension patients with irreversible pulmonary vascular disease from those whose PAP normalized after ventricular septal defect closure, plots of RC against Qp/Qs clearly distinguished the two groups [5].

\subsubsection{Right Ventricular Function and Coupling to PA Load}

In addition to the precise assessment and understanding of pathophysiological changes in the pulmonary vascular bed as shown thus far, an accurate assessment of intrinsic right ventricular (RV) contractility and its relation to the pulmonary artery (PA) load is essential for refining risk stratification and optimizing treatment in pulmonary hypertension because prognosis in PAH is now known to be strongly related to RV compensation rather than to the degree of the vascular injury itself as reported by many investigators [6-8]. Therefore, we will briefly introduce the method of assessing RV-PA interaction or RV-PA coupling. 
The ventricular pressure-volume relationship is the best way to demonstrate this [9-11]. An instantaneous plot of ventricular pressure and volume change yields a pressure-volume loop [12]. We can further construct successive ventricular pressure-volume loops during transient preload reduction with inferior vena cava occlusion $[11,13,14]$. The slope of the end-systolic pressure-volume relationship, called end-systolic elastance (Ees), represents ventricular contractility, whereas arterial elastance (Ea) represents ventricular afterload and provides information on PA impedance $[14,15]$. Therefore, the simple ratio of Ees/Ea represents ventricular-arterial (V-A) coupling status [16]. Figure 15.7a shows the PV relationship in the normal left ventricle (LV). Compared to that for the LV, the normal RV pressure-volume relationship has, as shown in Fig. 15.7b, a triangular shape with a low ventricular contractility corresponding to the low PA impedance or low ventricular afterload. With progression of pulmonary hypertension, the RV generally adapts to increased afterload by increasing contractility, preserving V-A coupling status and, thereby, cardiac output (Fig. 15.8a, b). However, once the RV contractile adaptation begins to fail, the RV needs to increase its volume to preserve cardiac output, leading to a course of progressive failure and poor outcome as shown in the yellow lines. The PV relationship is also useful to predict treatment response. Because Ees represents ventricular systolic stiffness, it predicts pressure variation at a given change in loading condition and, thereby, treatment response $[17,18]$.

a

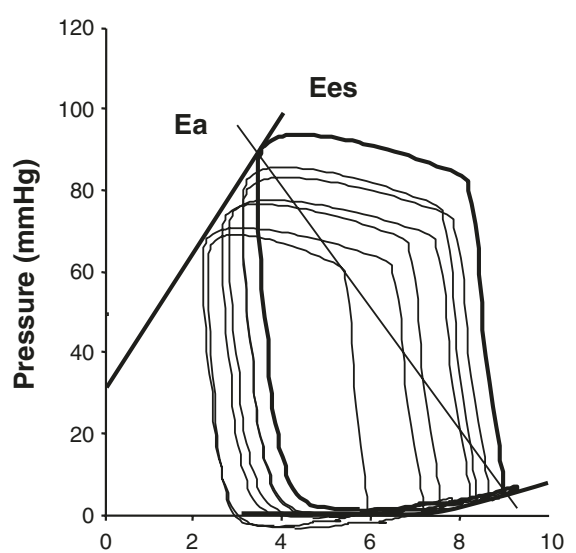

b

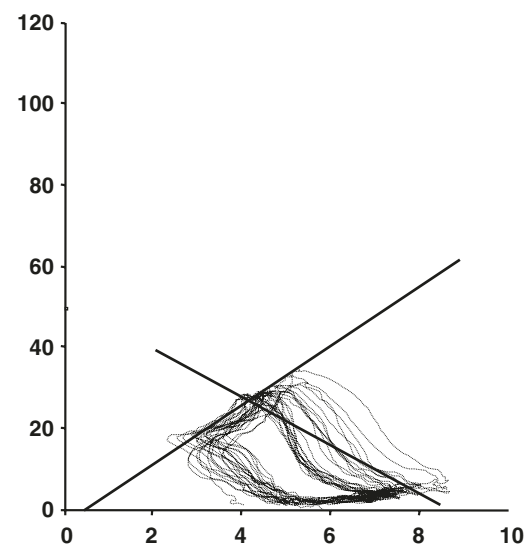

Fig. 15.7 Examples of pressure-volume relationships in normal left ventricle (a) and right ventricle $(\mathbf{b})$ 

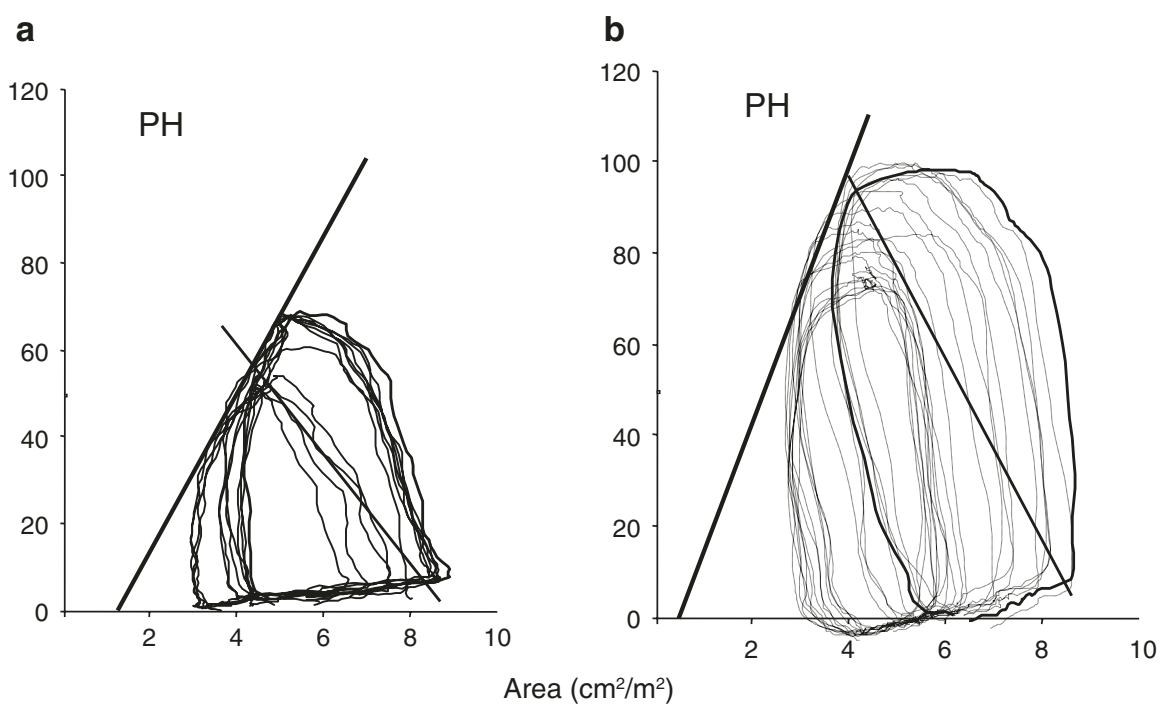

Afterload mismatch

RV dysfunction

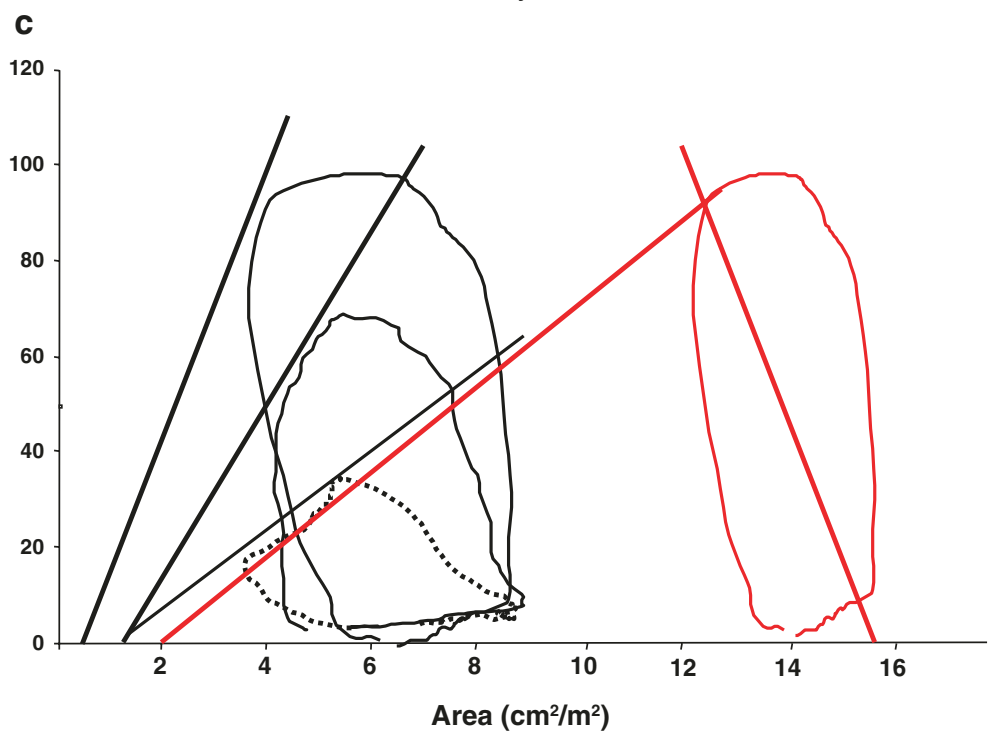

Fig. 15.8 Changes in pressure-volume relationships with progression $(\mathbf{a}, \mathbf{b})$ of pulmonary hypertension $(\mathrm{PH})$. Afterload-mismatched right ventricle $(\mathrm{RV})$ represented by red lines $(\mathbf{c})$ 


\subsubsection{Abnormalities of Compliance Is the Main Pathophysiology}

Next, we will discuss another disease entity of abnormal pulmonary circulation in which change in arterial compliance is the predominant pathophysiology of pulmonary hypertension. Tetralogy of Fallot (TOF) and multiple peripheral pulmonary stenosis are representative of this group. We measured pulmonary vascular impedance in 29 pediatric patients with repaired TOF [19]. Impedance spectra of TOF shifted upward compared to those of control patients, and PA exhibited augmented pulse pressure with increased wave reflection [19]. As summarized in Fig. 15.9, the abnormality of pulmonary vasculature in TOF is more evident in elastic components than in resistive components as shown by the increased characteristic impedance and decreased arterial compliance. Wave reflection is also augmented, contributing to the change in pulsatile nature of the pulmonary circulation in TOF. These data indicating enhanced pulmonary arterial stiffness are consistent with a previous histologic examination of the pulmonary artery in patients with TOF [20, 21]. Bedard et al. reported abnormal elastic tissue configuration and a high proportion of medionecrosis and elastic fragmentation [20]. These changes suggest decreased distensibility of the pulmonary arteries. Importantly, we also found that decreased pulmonary compliance was associated with RV dilation independent of the severity of pulmonary regurgitation [19]. Therefore, decreased pulmonary compliance in TOF serves as a pulsatile afterload to the RV and contributes to the progression of RV failure.

\subsubsection{Non-pulsatile Pulmonary Flow Is the Main Pathophysiology}

Lastly, cavo-pulmonary connection, as in Fontan or Glenn circulation, is another disease entity of abnormal pulmonary circulation in congenital heart disease. Although Fontan circulation does not cause apparent pulmonary hypertension,
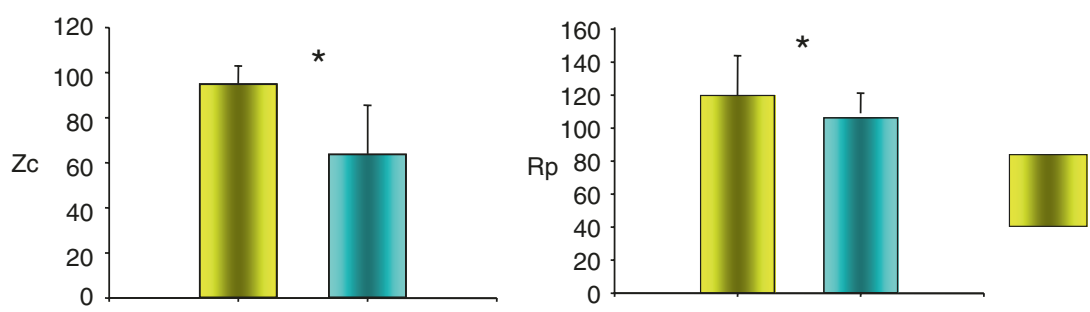
TOF
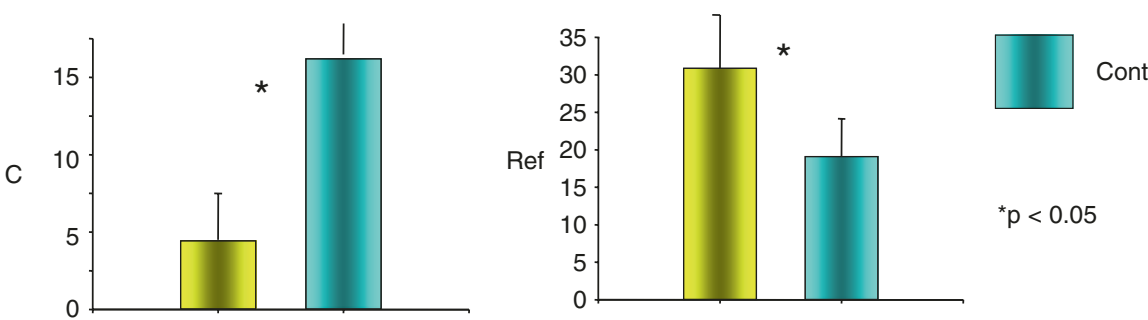

${ }^{*} p<0.05$

Fig. 15.9 Comparison of characteristic impedance (Zc), pulmonary resistance (Rp), compliance (C), and wave reflection (Ref) between tetralogy of Fallot (TOF) and control patients (Cont) 
unique pulmonary hemodynamics are characterized by non-pulsatile flow due to lack of a pulmonary ventricle [22]. The pulsatile nature of pulmonary blood flow is important for shear stress-mediated release of endothelium-derived nitric oxide (NO) and lowering of PVR, thereby reducing NO-cGMP-mediated vasodilatory capacity [23-25]. In addition, low cardiac output and elevated central venous pressure (CVP) in Fontan circulation can activate vasoconstrictive hormones, including endothelin and angiotensin II [22, 26, 27]. Therefore, PVR is generally slightly but significantly higher than normal even in good Fontan status. In fact, we can decrease PVR using exogenous NO in patients late after Fontan surgery. Redington's group examined NO responsiveness in 15 Fontan patients with a median postoperative period of 9 years and demonstrated significant reduction of PVR with inhaled NO. [28] Interestingly, the effect was even more evident in patients with total cavopulmonary connection (TCPC) than in those with atrio-pulmonary connection (APC) and some degree of pulmonary flow pulsatility, suggesting the importance of pulsatility [28]. Our own data also indicated significant reduction of PAP using sildenafil or tadalafil in 26 patients with Glenn circulation (Fig. 15.10).

There are many studies indicating activation of endothelin-1 or the reninangiotensin system in Fontan circulation [29-31]. A significant correlation between endothelin-1 and PVR after the Fontan operation was also reported [31]. In addition, an endothelin receptor antagonist effectively reduced PVR in Fontan patients and improved exercise tolerance and quality of life [32]. Therefore, even in good Fontan status, pulmonary dilators targeting NO and endothelin pathways may have some role in improving Fontan pulmonary circulation and thereby improving the long-term outcome.

Let us briefly think about the role of pulmonary compliance or capacitance in the Fontan circulation. Theoretically, as we explained earlier, only pulsatile flow goes into capacitance, and thus, in the Fontan pulmonary circulation where pulmonary flow is basically non-pulsatile, PAP is determined predominantly by the PVR [22].

Fig. 15.10 Changes in pulmonary artery pressure (PAP) before and after oral administration of sildenafil in Glenn patients

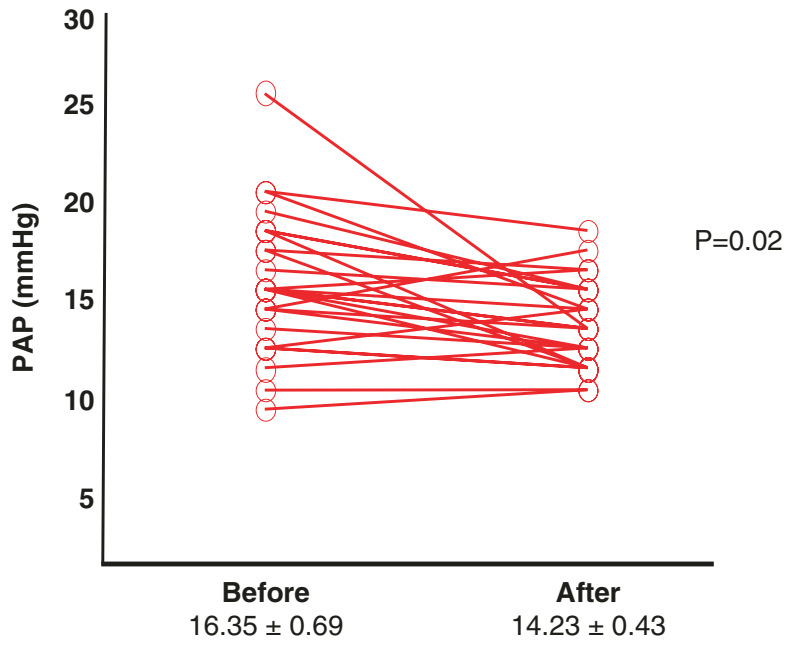


Nonetheless, pulmonary vascular capacitance still possesses important pathophysiological roles in the Fontan circulation. First, because of the direct connection of systemic and pulmonary circulation, pulmonary vascular capacitance serves as part of the total vascular impedance from the aorta to the pulmonary vascular bed [22]. Total vascular impedance is afterload to the Fontan single ventricle. As shown here, total vascular impedance, or ventricular afterload, increases with an increase in pulmonary vascular capacitance [22]. Second, perhaps more importantly, our computer simulation clearly indicated that smaller pulmonary vascular capacitance is related to a more marked increase in CVP in response to changes in pulmonary blood volume, which can occur with exercise and excessive water intake [33, 34]. Therefore, CVP variation during daily life should be pronounced in Fontan patients with decreased pulmonary capacitance. As in the original "10 Commandments" or indications for Fontan surgery [35], pulmonary arterial size, which is related to pulmonary capacitance, is important, independent of PVR, for the establishment of stable Fontan hemodynamics.

Similar to the effects of pulmonary artery capacitance, systemic venous capacitance is important and is an even more important hemodynamic parameter to establish lower levels of CVP in the Fontan circulation $[34,36]$.

Figure 15.11 shows the effects of changes in each vascular parameter in Fontan hemodynamics [33]. Each parameter was changed by $50 \%$ from the baseline values and their effects on CVP were examined by computer simulation. As shown, venous capacitance has the strongest influence on the change in CVP, followed by pulmonary capacitance and pulmonary resistance [33]. The importance of venous capacitance is realized by our "super-Fontan" strategy, which is an aggressive venodilation therapy with nitrates and angiotensin-converting enzyme (ACE) inhibitors, and pulmonary dilators if necessary, to achieve supernormal, extremely good Fontan circulation [37]. In fact, this strategy significantly increases venous capacitance as measured by the dye-dilution technique [37-39]. As shown in Fig. 15.11, eight consecutive patients who received super-Fontan therapy soon after Fontan surgery had

Fig. 15.11 The effects of changes in each vascular parameter in Fontan. Cs systemic venous compliance, $R s$ systemic resistance, $R p$ pulmonary resistance, $C p$ pulmonary compliance

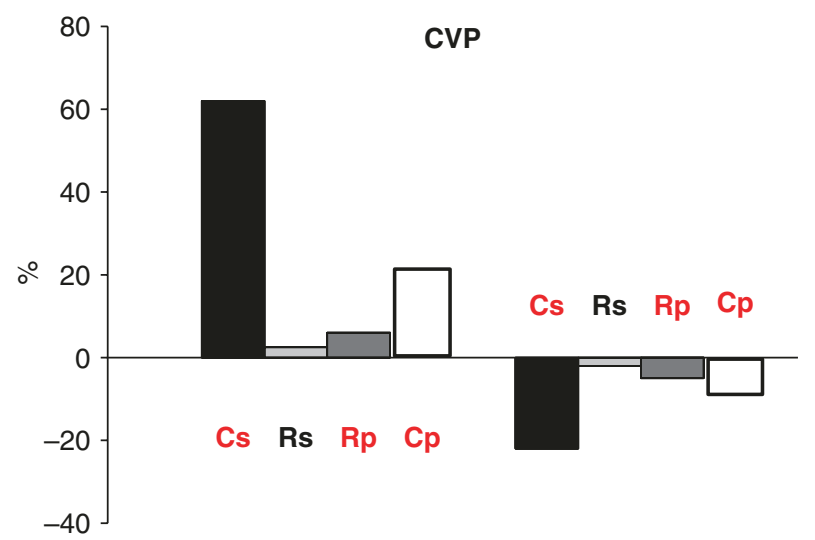


significantly lower CVP values than patients without super-Fontan strategy at cardiac catheterization 1 year after Fontan surgery; however, the cardiac index (CI) was comparable in both groups. Six patients who received super-Fontan therapy had CVP values of $<10 \mathrm{mmHg}$.

In addition, we newly applied this strategy to 20 patients at an average 7 years postoperatively. The venous capacitance measured by the dye-dilution technique significantly increased after 6 months of this therapy. Importantly, with this change in venous capacitance, CVP again significantly and consistently decreased after the therapy without changes in heart rate and cardiac index.

We will close this chapter by presenting a case of protein-losing enteropathy (PLE) in which super-Fontan strategy was very effective. The patient was a 16-year-old boy with asplenia syndrome, major atrio-pulmonary collateral arteries, and atrioventricular valve regurgitation who underwent TCPC at the age of 7 but developed PLE after the TCPC. Every possible medication had been prescribed. He was referred to our hospital and underwent the super-Fontan strategy, which effectively reduced CVP from 21 to $15 \mathrm{mmHg}$ and, more importantly, resolved the PLE.

In summary, comprehensive assessment of changes in the pulmonary vascular bed and their coupling to RV function is important. Impedance analysis and determination of pressure-volume relationships are ideal methods for this purpose. There are several disease entities with abnormal pulmonary circulation in congenital heart disease characterized by abnormalities of resistance, compliance, and flow pulsatility. Venous capacitance as well as pulmonary resistance can be an important therapeutic target to improve Fontan prognosis.

\section{References}

1. Murgo JP, Westerhof N, Giolma JP, Altobelli SA. Aortic input impedance in normal man: Relationship to pressure wave forms. Circulation. 1980;62:105-16.

2. Senzaki H, Chen CH, Ishido H, Masutani S, Matsunaga T, Taketazu M, Kobayashi T, Sasaki N, Kyo S, Yokote Y. Arterial hemodynamics in patients after Kawasaki disease. Circulation. 2005;111:2119-25.

3. Newman DL, Walesby RK, Bowden NL. Hemodynamic effects of acute experimental aortic coarctation in the dog. Circ Res. 1975;36:165-72.

4. Senzaki H, Isoda T, Ishizawa A, Hishi T. Reconsideration of criteria for the fontan operation. Influence of pulmonary artery size on postoperative hemodynamics of the fontan operation. Circulation. 1994;89:1196-202.

5. Senzaki H, Kato H, Akagi M, Hishi T, Yanagisawa M. New criteria for the radical repair of congenital heart disease with pulmonary hypertension. In order to avoid postoperative residual pulmonary hypertension. Jpn Heart J. 1995;36:49-59.

6. Vanderpool RR, Pinsky MR, Naeije R, Deible C, Kosaraju V, Bunner C, Mathier MA, Lacomis J, Champion HC, Simon MA. Rv-pulmonary arterial coupling predicts outcome in patients referred for pulmonary hypertension. Heart. 2015;101:37-43.

7. Inuzuka R, Kass DA, Senzaki H. Novel, single-beat approach for determining both endsystolic pressure-dimension relationship and preload recruitable stroke work. Open Heart. 2016;3:e000451. 
8. Sugimoto M, Masutani S, Seki M, Kajino H, Fujieda K, Senzaki H. High serum levels of procollagen type III-n-terminal amino peptide in patients with congenital heart disease. Heart. 2009;95(24):2023-8.

9. Senzaki H, Naito C, Masutani S, Nogaki M, Ohono A, Kobayashi J, Sasaki N, Asano H, Shunei K, Yokote Y, Kobayashi T. Hemodynamic evaluation for closing interatrial communication after fenestrated fontan operation. J Thorac Cardiovasc Surg. 2001;121:1200-2.

10. Senzaki H, Chen CH, Masutani S, Taketazu M, Kobayashi J, Kobayashi T, Sasaki N, Asano H, Kyo S, Yokote Y. Assessment of cardiovascular dynamics by pressure-area relations in pediatric patients with congenital heart disease. J Thorac Cardiovasc Surg. 2001;122:535-47.

11. Senzaki H, Chen H, Kass D. Single-beat estimation of end-systolic pressure-volume relation in humans a new method with the potential for noninvasive application. Circulation. 1996;94:2497-506.

12. Senzaki H, Gluzband YA, Pak PH, Crow MT, Janicki JS, Kass DA. Synergistic exacerbation of diastolic stiffness from short-term tachycardia-induced cardiodepression and angiotensin ii. Circ Res. 1998;82:503-12.

13. Senzaki H, Miyagawa K, Kishigami Y, Sasaki N, Masutani S, Taketazu M, Kobayashi J, Kobyashi T, Asano H, Kyo S, Yokote Y. Inferior vena cava occlusion catheter for pediatric patients with heart disease: For more detailed cardiovascular assessments. Catheter Cardiovasc Interv. 2001;53:392-6.

14. Senzaki H, Isoda T, Paolocci N, Ekelund U, Hare JM, Kass DA. Improved mechanoenergetics and cardiac rest and reserve function of in vivo failing heart by calcium sensitizer emd-57033. Circulation. 2000;101:1040-8.

15. Senzaki H, Masutani S, Ishido H, Taketazu M, Kobayashi T, Sasaki N, Asano H, Katogi T, Kyo $\mathrm{S}$, Yokote Y. Cardiac rest and reserve function in patients with fontan circulation. J Am Coll Cardiol. 2006;47:2528-35.

16. Senzaki H, Fetics B, Chen CH, Kass DA. Comparison of ventricular pressure relaxation assessments in human heart failure: Quantitative influence on load and drug sensitivity analysis. J Am Coll Cardiol. 1999;34:1529-36.

17. Senzaki H, Iwamoto Y, Ishido H, Masutani S, Taketazu M, Kobayashi T, Katogi T, Kyo $\mathrm{S}$. Ventricular-vascular stiffening in patients with repaired coarctation of aorta: Integrated pathophysiology of hypertension. Circulation. 2008;118:S191-8.

18. Khono K, Tamai A, Kobayashi T, Senzaki H. Effects of stent implantation for peripheral pulmonary artery stenosis on pulmonary vascular hemodynamics and right ventricular function in a patient with repaired tetralogy of fallot. Heart Vessels. 2011;26(6):672-6.

19. Inuzuka R, Seki M, Sugimoto M, Saiki H, Masutani S, Senzaki H. Pulmonary arterial wall stiffness and its impact on right ventricular afterload in patients with repaired tetralogy of fallot. Ann Thorac Surg. 2013;96:1435-41.

20. Bedard E, McCarthy KP, Dimopoulos K, Giannakoulas G, Gatzoulis MA, Ho SY. Structural abnormalities of the pulmonary trunk in tetralogy of fallot and potential clinical implications: A morphological study. J Am Coll Cardiol. 2009;54:1883-90.

21. Senzaki H, Iwamoto Y, Ishido H, Matsunaga T, Taketazu M, Kobayashi T, Asano H, Katogi T, Kyo S. Arterial haemodynamics in patients after repair of tetralogy of fallot: Influence on left ventricular after load and aortic dilatation. Heart. 2008;94:70-4.

22. Senzaki H, Isoda T, Ishizawa A, Hishi T. Reconsideration of criteria for the fontan operation. Influence of pulmonary artery size on postoperative hemodynamics of the fontan operation. Circulation. 1994;89:266-71.

23. Recchia FA, Senzaki H, Saeki A, Byrne BJ, Kass DA. Pulse pressure-related changes in coronary flow in vivo are modulated by nitric oxide and adenosine. Circ Res. 1996;79:849-56.

24. Peng X, Haldar S, Deshpande S, Irani K, Kass DA. Wall stiffness suppresses akt/enos and cytoprotection in pulse-perfused endothelium. Hypertension. 2003;41:378-81.

25. Masutani S, Saiki H, Kurishima C, Ishido H, Tamura M, Senzaki H. Heart failure with preserved ejection fraction in children: Hormonal imbalance between aldosterone and brain natriuretic peptide. Circ J. 2013;77:2375-82. 
26. Ohuchi H, Takasugi H, Ohashi H, Yamada O, Watanabe K, Yagihara T, Echigo S. Abnormalities of neurohormonal and cardiac autonomic nervous activities relate poorly to functional status in fontan patients. Circulation. 2004;110:2601-8.

27. Saiki H, Kuwata S, Kurishima C, Iwamoto Y, Ishido H, Masutani S, Senzaki H. Aldosteronecortisol imbalance immediately after fontan operation with implications for abnormal fluid homeostasis. Am J Cardiol. 2014;114:1578-83.

28. Khambadkone S, Li J, de Leval MR, Cullen S, Deanfield JE, Redington AN. Basal pulmonary vascular resistance and nitric oxide responsiveness late after fontan-type operation. Circulation. 2003; 107:3204-8.

29. Ishida H, Kogaki S, Ichimori H, Narita J, Nawa N, Ueno T, Takahashi K, Kayatani F, Kishimoto H, Nakayama M, Sawa Y, Beghetti M, Ozono K. Overexpression of endothelin-1 and endothelin receptors in the pulmonary arteries of failed fontan patients. Int J Cardiol. 2012;159(1):34-9.

30. Inai K, Nakanishi T, Nakazawa M. Clinical correlation and prognostic predictive value of neurohumoral factors in patients late after the fontan operation. Am Heart J. 2005;150:588-94.

31. Hiramatsu T, Imai Y, Takanashi Y, Seo K, Terada M, Aoki M, Nakazawa M. Time course of endothelin-1 and adrenomedullin after the fontan procedure. Ann Thorac Surg. 1999;68:169-72.

32. Agnoletti G, Gala S, Ferroni F, Bordese R, Appendini L, Pace Napoleone C, Bergamasco L. Endothelin inhibitors lower pulmonary vascular resistance and improve functional capacity in patients with fontan circulation. J Thorac Cardiovasc Surg. 2017;153:1468-75.

33. Liang F, Senzaki H, Yin Z, Fan Y, Sughimoto K, Liu H. Transient hemodynamic changes upon changing a bcpa into a tcpc in staged fontan operation: A computational model study. ScientificWorldJournal. 2013;2013:486815.

34. Liang F, Senzaki H, Kurishima C, Sughimoto K, Inuzuka R, Liu H. Hemodynamic performance of the fontan circulation compared with a normal biventricular circulation: A computational model study. Am J Physiol. 2014;307:H1056-72.

35. Fontan F, Baudet E. Surgical repair of tricuspid atresia. Thorax. 1971;26:240-8.

36. Saiki H, Sugimoto M, Kuwata S, Kurishima C, Iwamoto Y, Ishido H, Masutani S, Senzaki H. Novel mechanisms for cerebral blood flow regulation in patients with congenital heart disease. Am Heart J. 2016;172:152-9.

37. Kurishima C, Saiki H, Masutani S, Senzaki H. Tailored therapy for aggressive dilatation of systemic veins and arteries may result in improved long-term fontan circulation. J Thorac Cardiovasc Surg. 2015;150(5):1367-70.

38. Masutani S, Kurishima C, Yana A, Kuwata S, Iwamoto Y, Saiki H, Ishido H, Senzaki $\mathrm{H}$. Assessment of central venous physiology of fontan circulation using peripheral venous pressure. J Thorac Cardiovasc Surg. 2017;153:912-20.

39. Kim J, Kuwata S, Kurishima C, Iwamoto Y, Ishido H, Masutani S, Senzaki H. Importance of dynamic central venous pressure in fontan circulation. Heart Vessels. 2018;33(6):664-70.

Open Access This chapter is licensed under the terms of the Creative Commons Attribution 4.0 International License (http://creativecommons.org/licenses/by/4.0/), which permits use, sharing, adaptation, distribution and reproduction in any medium or format, as long as you give appropriate credit to the original author(s) and the source, provide a link to the Creative Commons license and indicate if changes were made.

The images or other third party material in this chapter are included in the chapter's Creative Commons license, unless indicated otherwise in a credit line to the material. If material is not included in the chapter's Creative Commons license and your intended use is not permitted by statutory regulation or exceeds the permitted use, you will need to obtain permission directly from the copyright holder.

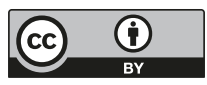

